\section{Damaged muscle fibers might masquerade as hybrid fibers - a cautionary note on immunophenotyping mouse muscle with mouse monoclonal antibodies}

\author{
Morium Begam, Joseph A. Roche \\ Department of Health Care Sciences, \\ Physical Therapy Program, Wayne State \\ University, Detroit MI, USA
}

\begin{abstract}
We report that, labeling mouse muscle tissue, with mouse monoclonal antibodies specific to slow or fast myosin heavy chain (sMyHC and $\mathrm{fMyHC}$, respectively), can lead to artefactual labeling of damaged muscle fibers, as hybrid fibers ( $\mathrm{sMHC}+$ and $\mathrm{fMyHC}+$ ). We demonstrate that, such erroneous immunophenotyping of muscle may be avoided, by performing colabeling or serialsection-labeling, to identify damaged fibers. The quadriceps femoris muscle group $(\mathrm{QF})$ in 7-month-old, male, C57BL/6J mice had: $1.21 \pm 0.21 \%, 98.34 \pm 1.06 \%, 0.07 \pm 0.01 \%$, and $0.53 \pm 0.85 \%$ fibers, that were, $\mathrm{sMyHC}+$, $\mathrm{fMyHC}+$, hybrid, and damaged, respectively. All fibers in the tibialis anterior muscle (TA) of 3-month-old, male, C57BL/6J mice were $\mathrm{fMyHC}+$; and at 3 days after injurious eccentric contractions, there was no fiber-type shift, but $\sim 18 \%$ fibers were damaged.
\end{abstract}

\section{Introduction}

Skeletal muscle tissue possesses the unique property of being able to generate active contractile force for voluntary movement. Muscle contraction is made possible by cyclic interaction of myosin heavy chains (MyHCs) in the thick filaments of sarcomeres with actin in thin filaments, through the hydrolysis of ATP. ${ }^{1,2}$ Movement is mediated by the activation of motor units, which are made up of all muscle fibers that receive efferent input from a single motor axon. $^{3,4}$ Depending on whether muscle fibers are part of a fast or slow motor unit, they possess slow MyHC (sMyHC, a.k.a. type $1 \mathrm{MyHC}$ ) or fast MyHC (fMyHC, a.k.a. type $2 \mathrm{MyHC}$; subtypes $2 \mathrm{~A}$ and $2 \mathrm{X}$ in humans, along with $2 \mathrm{~B}$ in other mammalian species). , $5,6^{2}$ Immunophenotyping of muscles, based on fiber type, is possible due to the availability of antibodies against specif- ic MyHC isoforms. ${ }^{6-8}$ Either naturally, or due to genetic/epigenetic influences, muscles may have a population of hybrid fibers, which have more than one type of MyHC.,, 10 Based on an incidental finding in our laboratory, we developed a set of experiments, to test the hypothesis that damaged muscle fibers label falsely as hybrid fibers. In order to test our hypothesis, we studied the quadriceps femoris (QF) muscle group of healthy middle-aged mice (7 months), since the QF in some of these mice shows small amounts of spontaneous damage. Also, since, intense eccentric muscle contractions are known to cause muscle fiber necrosis by disrupting sarcomeres, the sarcolemma, and the excitation-contraction coupling apparatus; we exposed the tibialis anterior (TA) muscle of healthy young mice ( 3 months) to a bout of medium-strain forced eccentric exercise (MSFEE), to assess if experimentally-injured muscle fibers might also present as hybrid fibers. ${ }^{11-16}$

\section{Materials and Methods}

\section{Animal models}

Experiments with live animals were performed at Wayne State University (Detroit, MI), according to protocols approved by the Institutional Animal Care and Use Committee. These protocols were in accordance with the Guide for the Care and Use of Laboratory Animals (1996, published by National Academy Press, 2101 Constitution Ave. NW, Washington, DC). All mice in this study were from the C57BL/6J strain (Stk\# 664, The Jackson Laboratory, Bar Harbor, ME), males, 7 month-old, for studies on the QF $(n=3$ mice), and 3 month-old, for studies on the TA ( $n=3$ mice $)$.

\section{Tissue collection}

We removed the QF and TA en bloc from euthanized mice, by cutting the distal tendon, reflecting the muscle upward with tweezers, and then releasing the proximal attachments with a scalpel. We quickly dipped the harvested muscles in mineral oil for cryoprotection, blotted of the excess oil with lab wipes, placed the muscles on aluminum foil, and snap froze them by rapidly immersing them in liquid nitrogen.

\section{Histological studies}

A brief description of our immunolabeling protocols is provided here; additional details are provided under Supplementary Methods and Supplementary Data.
Correspondence: Joseph A. Roche, Physical Therapy Program, Department of Health Care Sciences, Wayne State University, 259 Mack Ave., Room 4440, Detroit, MI 48201, USA. Tel. +1.313.577-1432.

E-mail: joseph.roche@wayne.edu

Key words: Skeletal muscle; myosin heavy chains; fiber type; immunolabeling; hybrid muscle fibers; muscle fiber damage; muscle injury.

Contributions: MB, JAR, collected and analyzed the data, and co-wrote the manuscript.

Conflict of interest: The authors declare no conflict of interest to disclose.

Acknowledgments: This work was made possible through a Pilot Grant from the Alliance for Regenerative Rehabilitation Research and Training (AR3T), NIH 1R03HD091648-01 from NICHD, a grant from the Jain Foundation Inc., a Faculty Research Awards Program (FRAP) grant from EACPHS Wayne State University, and a faculty startup package from Wayne State University, to JAR. AR3T is supported by the Eunice Kennedy Shriver National Institute of Child Health and Human Development (NICHD), National Institute of Neurological Disorders and Stroke (NINDS), and National Institute of Biomedical Imaging and Bioengineering (NIBIB) of the National Institutes of Health under Award Number P2CHD086843. The content of this paper is solely the responsibility of the authors and does not necessarily represent the official views of the National Institutes of Health. The authors would like to thank Renuka Roche, $\mathrm{PhD}$, OTR/L, Assistant Professor in Occupational Therapy, Eastern Michigan University, for critically reading and editing this manuscript.

Received for publication: 5 February 2018 . Accepted for publication: 6 July 2018.

This work is licensed under a Creative Commons Attribution-NonCommercial 4.0 International License (CC BY-NC 4.0).

(C) Copyright M. Begam and J.A. Roche, 2018 Licensee PAGEPress, Italy

European Journal of Histochemistry 2018; 62:2896 doi:10.4081/ejh.2018.2896

\section{MyHC labeling on QF muscle sections from 7-month-old mice}

We followed the labeled streptavidin biotin (LSAB) method to label MyHC and visualize it under confocal optics. ${ }^{(17)} \mathrm{We}$ incubated serial sections overnight, at $4{ }^{\circ} \mathrm{C}$, separately, with mouse monoclonal primary antibodies specific to sMyHC or $\mathrm{fMyHC}$ (M8421 and M4276, respectively, 1:1000, MilliporeSigma, St. Louis, MO, USA). ${ }^{18}$ 
After washing off unbound primary antibodies, sections were incubated for $60 \mathrm{~min}$ with secondary antibodies conjugated to biotin (goat-anti-mouse IgG, B2763, 1:200, ThermoFisher Scientific, Waltham, MA, USA). After washing off unbound secondary antibodies, we incubated sections for 15 min with streptavidin conjugated to Alexa 568 (S11226, 1:200, ThermoFisher Scientific).

\section{Desmin colabeling on QF muscle sections} from 7-month-old mice

Since desmin is highly sensitive to muscle fiber damage, we colabeled QF sections with rabbit polyclonal primary antibodies to desmin (RB-9014-P, 1:200, ThermoFisher Scientific, Waltham, MA), to detect damaged fibers in the QF. ${ }^{19,20}$ After overnight incubation and washing, as above; we incubated sections with secondary antibodies conjugated to Alexa 488 for 60 min (goat-
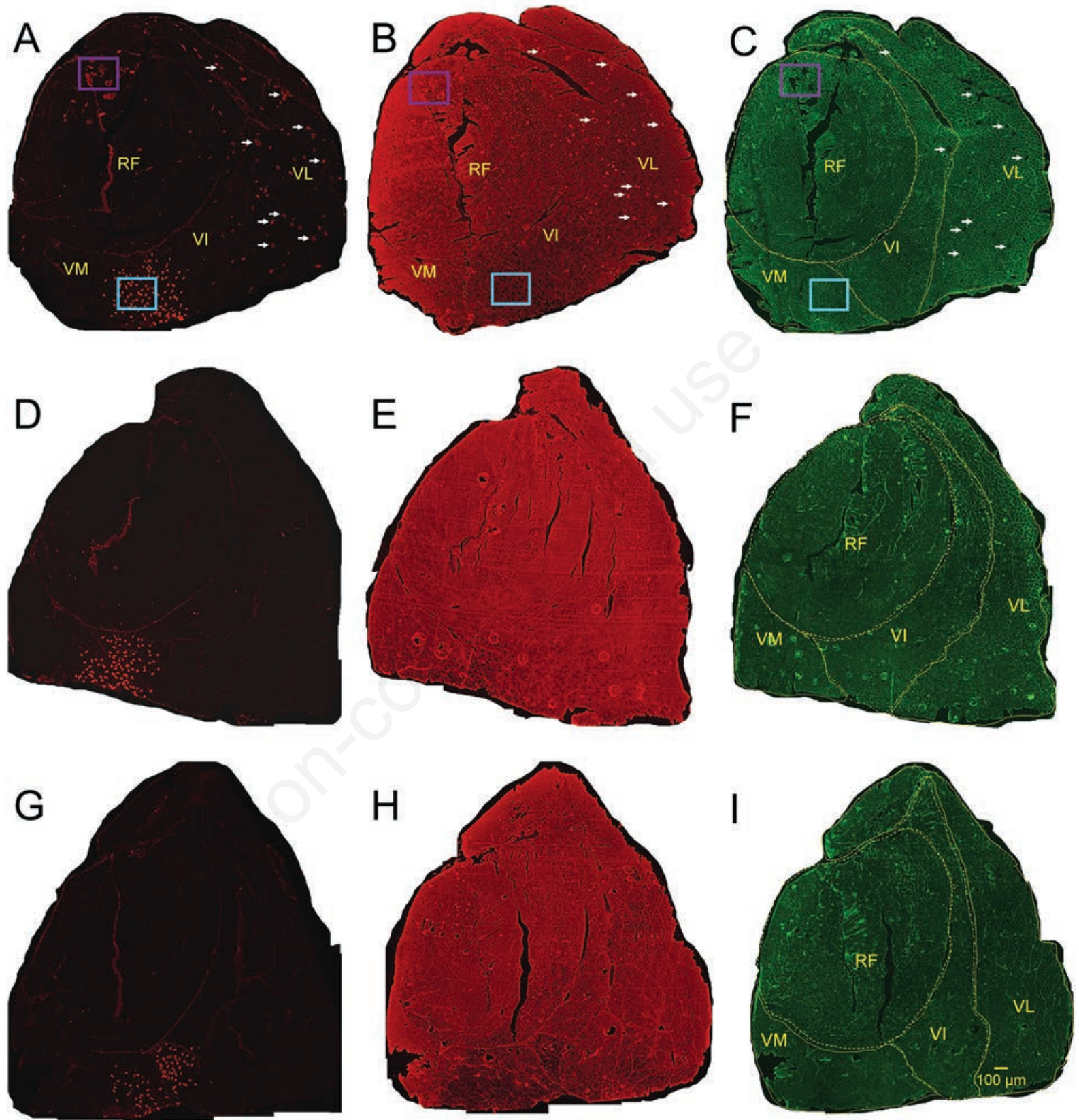

Figure 1. $\mathrm{fMyHC}$ + fibers predominate in the QF of 7-month-old mice with some sMyHC+ fibers in the VM and VI. This figure shows tiled images of serial or colabeled cross sections of the QF muscle group, from 7-mo-old C57BL/6J mice ( $\mathrm{n}=3$; A-C, D-F, and G-I are from animals 1,2 , and 3, respectively). We labeled sections with antibodies to sMyHC $(\mathrm{A}, \mathrm{D}, \mathrm{G})$ or $\mathrm{fMyHC}(\mathrm{B}, \mathrm{E}, \mathrm{H})$, and colabeled sections with antibodies to desmin to detect muscle fiber damage $(\mathrm{C}, \mathrm{F}, \mathrm{I})$. In all 3 mice, the QF was mostly composed of $\mathrm{fMyHC}+$ fibers, except for a small number of $s M y H C$ + fibers that were concentrated in the $V M$ and $V I$ portions of the $Q F$ (A-C, region in blue boxes; high magnification images are shown in Figure 2). In 1 of 3 mice (A-C), we found several fibers across the entire QF, which appeared to be sMyHC+ and fMyHC+ (white arrows); however, these fibers were desmin-, and were therefore damaged fibers and not hybrid fibers (region in purple boxes; high magnification images are shown in Figure 2). 

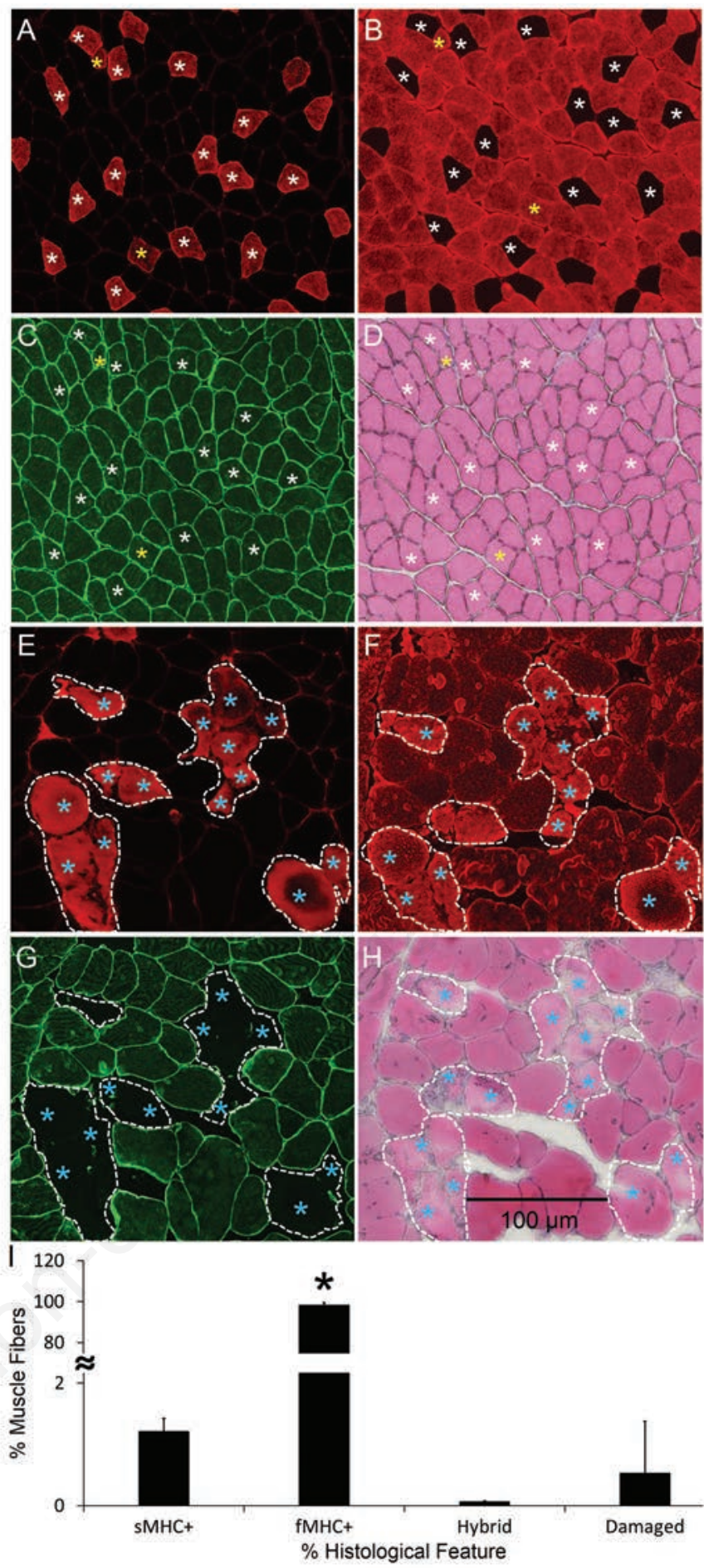

Figure 2. Damaged muscle fibers in the QF of 7-month-old mice appear as hybrid fibers. Panels A, B, and C, are images from the QF of animal 1, enclosed within the blue box in Figure $1 \mathrm{~A}-\mathrm{C}$, and are results of immunostaining with antibodies to $\mathrm{sMyHC}$, $\mathrm{fMyHC}$, and desmin, respectively. H\&E staining of the same region in A-C, is shown in D. The data in A-D suggest that, even though the VM and VI portions of the QF have sMyHC+ fibers, there are very few hybrid fibers. The white asterisks in A-D, denote the same set of $s M y H C+$ (i.e. $\mathrm{fMyHC}$-) fibers in serial sections. The yellow asterisks in $\mathrm{A}-\mathrm{D}$, denote hybrid fibers that are $\mathrm{MyHC}_{+}, \mathrm{fMyHC}_{+}, \mathrm{desmin}+$, and show no damage through H\&E staining. E, F, and G, are images from the QF of animal 1, enclosed within the purple box in Figure 1 A-C, and are results of immunostaining with antibodies to $s \mathrm{MyHC}, \mathrm{fMyHC}$, and desmin, respectively. $\mathrm{H} \& \mathrm{E}$ staining of the same region in $\mathrm{E}-\mathrm{G}$, is shown in $\mathrm{H}$. In panels E-H, damaged regions are enclosed by white dashed lines, and damaged fibers are marked with blue asterisks. Damaged fibers are desmin- and show disruption seen through H\&E staining (pale and/or fragmented cytoplasm, with or without cellular infiltration). Damaged fibers appear to be positive for both $\mathrm{sMyHC}$ and fMyHC, likely due to entry of mouse IgG, which gets labeled by secondary antibodies used for $\mathrm{MyHC}$ labeling. Without verifying fiber integrity, damaged fibers, such as those shown in E-H, could be mistaken for hybrid fibers having both sMyHC and $\mathrm{fMyHC}$. Quantitative data from QF muscles of all three 7-month-old mice are summarized in panel I, and confirm that the percentage of $\mathrm{MyHC}+$ fibers is significantly greater than the percentage of $s \mathrm{MyHC}+$, hybrid and damaged fibers. ${ }^{*} \mathrm{P}<0.05$ for $\mathrm{fMyHC}+$ fibers versus other groups. Mean \pm SD. 
anti-rabbit $\mathrm{F}(\mathrm{ab}$ ')2 fragments, A-11070, 1:200, ThermoFisher Scientific).

\section{$H \& E$ staining on $Q F$ muscle sections} from 7-month-old mice

On serial sections, we performed hematoxylin and eosin (H\&E) staining, as described earlier and in Supplementary Methods. ${ }^{21,22}$

\section{MyHC labeling on TA muscle sections from 3-month-old mice}

We labeled TA muscle sections from 3month-old mice, just as we labeled QF sections from 7- month-old mice, but without colabeling desmin.

\section{Desmin and immunoglobulin G (IgG) labeling on TA muscle sections from 3-month-old mice}

On serial sections of the TA muscle, we labeled desmin and $\mathrm{IgG}$, to detect damaged fibers, as described earlier and in Supplementary Methods..$^{20,22}$ As above, we incubated sections overnight with primary antibodies to desmin, washed the sections, and then applied goat anti-rabbit secondary antibodies. While applying goat anti-rabbitsecondary antibodies, we simultaneously applied goat anti-mouse $\operatorname{IgG}$ antibodies conjugated to biotin, in order to label mouse $\mathrm{IgG}$. After washing off unbound secondary antibodies, we incubated sections for 15 min with streptavidin conjugated to Alexa 568 to visualize mouse IgG under confocal optics. By this method, damaged fibers are detected by their loss of desmin and inclusion of mouse IgG. ${ }^{19,20,23}$

\section{Dystrophin labeling on TA muscle sec- tions from 3-month-old mice}

On serial sections of the TA muscle, we labeled the sarcolemma-associated protein dystrophin, as an additional measure of muscle fiber damage. ${ }^{24}$ Labeling methods were similar to desmin labeling, above, albeit with rabbit polyclonal antibodies against dystrophin (RB-9024-P, 1:200, ThermoFisher Scientific).

\section{Negative control labeling}

For MyHC, desmin and dystrophin labeling experiments, we performed negative control labeling by replacing primary antibodies with equal concentrations of non-specific IgG from the host species. For IgG, we performed negative labeling by omitting goat anti-mouse IgG. Additional details are provided under Supplementary Methods and Supplementary Data.

\section{Muscle injury by eccentric contrac- tions}

To demonstrate that damaged fibers label falsely as hybrid fibers, we followed a protocol of injurious eccentric contractions, which has been described in detail. ${ }^{16} \mathrm{We}$ exposed the left TA to 40 eccentric contractions (under general anesthesia; inhaled, 2-
$5 \%$ for induction and $1-4 \%$ for maintenance), performed in 4 sets of 10 repetitions, with 2 min rest between sets. For each eccentric contraction, the dorsiflexors were tetanically stimulated, and the foot was plantarflexed from 90 to $160^{\circ}$, at $300 / \mathrm{S} .{ }^{16}$ The right TA served as an unexercised control.

\section{Statistical analyses}

We analyzed quantitative data with SigmaStat 3.5 software (Systat Software, San Jose, CA, USA). We counted all fibers in each QF and TA, and calculated the percentage of fibers in each muscle that were sMyHC+, fMyHC+, hybrid, or damaged. We analyzed fiber counts from the QF and TA, by one-way ANOVA. Where F-ratios were significant, post-hoc analyses with Holm-Sidak correction identified significant differences between groups. P-values $<0.05$ were considered significant. Data are reported as mean \pm standard deviation (SD).

\section{Results}

\section{Distribution of sMyHC+ and fMyHC+ fibers in the QF of 7- month-old C57BL/6J mice}

In the QF, $\sim 98 \%$ fibers were $\mathrm{fMyHC}+$ (Figures 1 and 2; 8783 \pm 372 fibers counted per $\mathrm{QF} ; \mathrm{n}=3$ mice). After identifying the rectus femoris (RF), vastus medialis (VM), vastus intermedius (VI), and vastus lateralis (VL) portions of the QF, based on prior data; we evaluated the distribution of sMyHC and $\mathrm{AMyHC}$ fibers in these individual portions of the QF. ${ }^{25,26}$ We found a concentration of $\mathrm{sMyHC}+$ fibers in the $\mathrm{VM}$ and VI (Figures 1 and 2). In the vicinity of the cluster of sMyHC+ fibers in the VM and VI, we found a very small number of hybrid fibers, which were sMyHC+, fMyHC+, desmin + , and showed no disruption through H\&E staining.

In one of the QF samples, we observed several fibers that appeared to be sMyHC+ and $\mathrm{fMyHC}+$, and were sporadically distributed across the entire QF (Figure $1 \mathrm{~A}-\mathrm{C}$, white arrows); however, these fibers showed a loss of desmin, and disruption through H\&E staining, confirming that they were damaged fibers and not hybrid fibers (Figures 1 and 2). As reported earlier, desmin labeling is seen internally in muscle fibers, with enrichment near the sarcolemma. ${ }^{20,27,28}$

\section{Distribution of sMyHC+ and fMyHC+ fibers in the TA of 3- month-old C57BL/6J mice}

In the control TA, all fibers assessed were $\mathrm{fMyHC}+$, with no detectable
sMyHC+, or hybrid fibers (Figure $3 \mathrm{~A}-\mathrm{C}$, G-I; $2113 \pm 80$ fibers counted per TA; $n=3$ ). At 3 days after injurious eccentric contractions, we did not detect a change in fiber type, but did see an increase in damaged fibers that were $\operatorname{IgG}+$, desmin-, dystrophin-, and showed disruption through $\mathrm{H} \& \mathrm{E}$ staining (Figure 3 D-F, J-L). As seen in the QF of 7-mo-old mice, damaged fibers appeared to be both $\mathrm{sMyHC}+$ and $\mathrm{AMyHC}+$, based on MyHC staining alone; but, the entry of $\mathrm{IgG}$, the loss of desmin and dystrophin, and fiber disruption seen through H\&E staining, confirmed that, these fibers were damaged fibers and not hybrid fibers.

\section{Discussion}

\section{Damaged fibers masquerade as hybrid fibers}

In 7-month-old mice, fMyHC+ fibers predominated in the QF, with a few sMyHC+ fibers in the VM and VI portions, and a very small number of hybrid fibers in the vicinity of sMyHC+ fibers. However, in 1 of 3 animals studied, we found what looked like hybrid fibers (both sMyHC+ and $\mathrm{fMyHC}+$ ) scattered across the entire cross sectional area of the QF. By labeling serial sections with antibodies to desmin and through H\&E staining, we confirmed that, damaged fibers in the QF, were appearing to be $\mathrm{sMHC}+$ and $\mathrm{fMyHC}+$. To empirically validate our observation that damaged fibers can present as hybrid fibers, we studied the TA in younger mice (3-monthold), after injurious eccentric contractions. We confirmed that, even in the experimentally-injured TA, MyHC labeling alone, gave the impression that damaged fibers were sMyHC+ and $\mathrm{fMyHC}+$. Since damaged fibers are permeable to native IgG (Figure $3 \mathrm{~F}, \mathrm{~J}-\mathrm{L}$ ), it is likely that damaged fibers showed artefactual labeling with the anti-mouse secondary antibodies used for MyHC labeling. ${ }^{20,22}$

\section{The topographical distribution of sMyHC+, fMyHC+, and hybrid fibers in the QF - a potential out- come measure}

Our data suggest that $\sim 98 \%$ of the QF in healthy, 7-month-old, C57BL/6J mice, is made up of $\mathrm{fMyHC}+$ fibers. However, we detected a cluster of sMyHC+ fibers in the $\mathrm{VM}$ and VI, and a very small number of hybrid fibers in the vicinity of sMyHC+ fibers. This topographical information, could serve as a potential outcome measure to study muscle pathology or adaptation (example: a shift in fiber type), and to preclinically evaluate experimental therapies (example: reversal of a fiber type shift). 

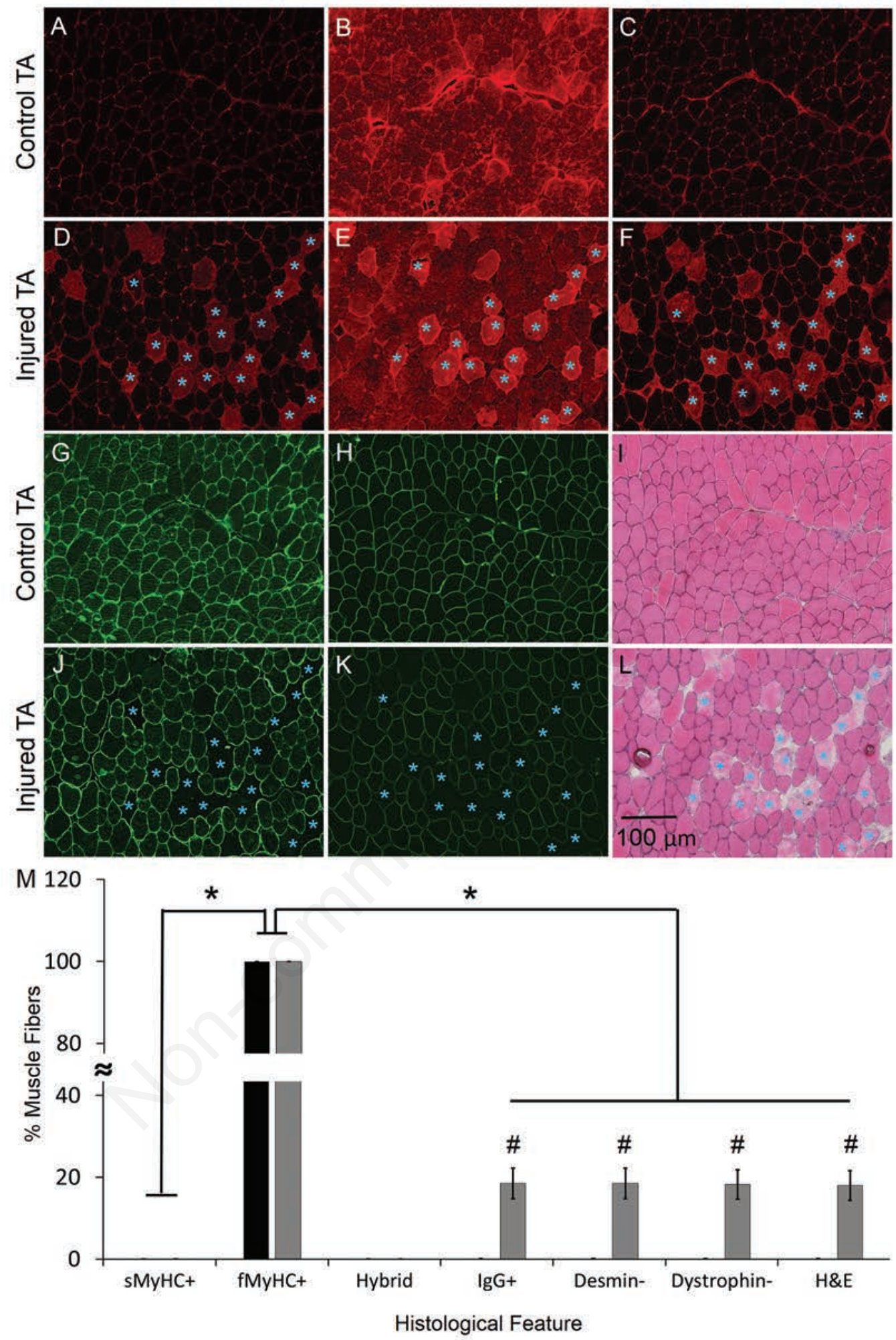

Figure 3. Representative serial sections of control TA muscle (A-C, G-I) and eccentrically-injured TA muscle (D-F, J-L, 3 days postinjury), are shown. Panels A, B, C, G and H, are labeled with antibodies to sMyHC, fMyHC, mouse IgG, desmin, and dystrophin, respectively; and so are $\mathrm{D}, \mathrm{E}, \mathrm{F}, \mathrm{J}$ and $\mathrm{K}$. Panels $\mathrm{I}$ and $\mathrm{L}$, are serial sections of control and injured TA, respectively, which are stained with H\&E. The data suggest that, all fibers in the control TA, are fMyHC+. Damaged fibers in the eccentrically-injured TA, can be identified by their loss of desmin and dystrophin, and inclusion of mouse IgG. Damaged fibers appear as if they are hybrid fibers, which are positive for both sMyHC and $\mathrm{AMHC}$ (marked with blue asterisks); however, this labeling is artefactual. Quantitative data are presented in panel $\mathrm{M}$. The data indicate that, the control TA is composed of $\mathrm{fMyHC}+$ fibers, and that there is no change in fiber type at 3 days after a single bout of injurious eccentric contractions. The black and grey bars in the graph, represent control and injured TA muscle, respectively. ${ }^{*} \mathrm{P}<0.05$ for $\mathrm{fMyHC}+$ fibers versus other groups; $\# \mathrm{P}<0.05$ for injured versus control TA. Mean $\pm \mathrm{SD}$. 


\section{Limitations of current study and alternative approaches}

Since muscle fibers can undergo segmental degeneration, studies such as ours, can only provide a snapshot of the status of a particular region, along the length of each muscle fiber observed in a cross section. Furthermore, artefactual MyHC labeling can be reduced by: using mouse-on-mouse labeling kits when using mouse monoclonal antibodies to label mouse tissue; using primary antibodies that have different immunoglobulin isoforms (example: IgG1, $\operatorname{IgG} 2 \mathrm{~b}, \operatorname{IgM})$; using antibodies raised in species other than the species being studied (example: avoiding mouse-on-mouse labeling); and, pre-conjugating primary antibodies to fluorophores before labeling., 29-31 Nonetheless, our data stress the importance of assessing fiber integrity, while immunophenotyping muscle based on MyHC isoforms.

Assessing myofiber integrity is a useful checkpoint, while immunophenotyping muscle for fiber-type distribution, based on MyHC isoforms.

\section{References}

1. Rassier DE. Sarcomere mechanics in striated muscles: from molecules to sarcomeres to cells. Am J Physiol Cell Physiol 2017;313:C134-45.

2. Scott W, Stevens J, Binder-Macleod SA. Human skeletal muscle fiber type classifications. Phys Ther 2001;81: 1810-6.

3. Buller AJ, Eccles JC, Eccles RM. Interactions between motoneurones and muscles in respect of the characteristic speeds of their responses. J Physiol 1960; 150:417-39.

4. Pette D, Vrbova G. The contribution of neuromuscular stimulation in elucidating muscle plasticity revisited. Eur J Transl Myol 2017;27:6368.

5. Schiaffino S, Reggiani C. Fiber types in mammalian skeletal muscles. Physiol Rev 2011;91:1447-531.

6. Schiaffino S. Muscle fiber type diversity revealed by anti-myosin heavy chain antibodies. Febs J 2018 doi: 10.1111/ febs. 14502 [Epub ahead of print].

7. Bloemberg D, Quadrilatero J. Rapid determination of myosin heavy chain expression in rat, mouse, and human skeletal muscle using multicolor immunofluorescence analysis. PLoS One 2012; 7:e35273.

8. Gregorevic P, Meznarich NA, Blankinship MJ, Crawford RW, Chamberlain JS.
Fluorophore-labeled myosin-specific antibodies simplify muscle-fiber phenotyping. Muscle Nerve 2008;37:104-6.

9. Hamalainen N, Pette D. Coordinated fastto-slow transitions of myosin and SERCA isoforms in chronically stimulated muscles of euthyroid and hyperthyroid rabbits. J Muscle Res Cell Motil 1997;18:545-54.

10. Matsuura T, Li Y, Giacobino JP, Fu FH, Huard J. Skeletal muscle fiber type conversion during the repair of mouse soleus: potential implications for muscle healing after injury. J Orthop Res 2007;25:153440.

11. Allen DG. Eccentric muscle damage: mechanisms of early reduction of force. Acta Physiol Scand 2001;171:311-9.

12. Proske U, Morgan DL. Muscle damage from eccentric exercise: mechanism, mechanical signs, adaptation and clinical applications. J Physiol 2001;537:333-45.

13. Lovering RM, Brooks SV. Eccentric exercise in aging and diseased skeletal muscle: good or bad? J Appl Physiol 2014;116: 1439-45.

14. Tidball JG. Mechanisms of muscle injury, repair, and regeneration. Compr Physiol. 2011;1:2029-62.

15. Lieber RL, Friden J. Mechanisms of muscle injury gleaned from animal models. Am J Phys Med Rehabil 2002;81(11 Suppl):S70-9.

16. Begam M, Collier AF, Mueller AL, Roche R, Galen SS, Roche JA. Diltiazem improves contractile properties of skeletal muscle in dysferlin-deficient BLAJ mice, but does not reduce contraction-induced muscle damage. Physiol Rep 2018;6: e13727.

17. Diamandis EP, Christopoulos TK. The biotin-(strept)avidin system: principles and applications in biotechnology. Clin Chem 1991;37:625-36.

18. Quattrocelli M, Swinnen M, Giacomazzi G, Camps J, Barthélemy I, Ceccarelli G, et al. Mesodermal iPSC-derived progenitor cells functionally regenerate cardiac and skeletal muscle. J Clin Invest 2015;125: 4463-82.

19. Barash IA, Peters D, Friden J, Lutz GJ, Lieber RL. Desmin cytoskeletal modifications after a bout of eccentric exercise in the rat. Am J Physiol Regul Integr Comp Physiol 2002;283:R958-63.

20. Begam M, Abro VM, Mueller AL, Roche JA. Sodium 4-phenylbutyrate reduces myofiber damage in a mouse model of Duchenne muscular dystrophy. Appl Physiol Nutr, Metab 2016;41:1108-11.

21. Roche JA, Ford-Speelman DL, Ru LW, Densmore AL, Roche R, Reed PW, et al. Physiological and histological changes in skeletal muscle following in vivo gene transfer by electroporation. Am J Physiol Cell Physiol 2011;301:C1239-50.

22. Roche JA, Tulapurkar ME, Mueller AL, van Rooijen N, Hasday JD, Lovering RM, et al. Myofiber damage precedes macrophage infiltration after in vivo injury in dysferlin-deficient $\mathrm{A} / \mathrm{J}$ mouse skeletal muscle. Am J Pathol 2015;185:1686-98.

23. Straub V, Rafael JA, Chamberlain JS, Campbell KP. Animal models for muscular dystrophy show different patterns of sarcolemmal disruption. J Cell Biol 1997; 139:375-85.

24. Lovering RM, De Deyne PG. Contractile function, sarcolemma integrity, and the loss of dystrophin after skeletal muscle eccentric contraction-induced injury. Am J Physiol Cell Physiol 2004;286:C230-8.

25. Talmadge RJ, Acosta W, Garland T, Jr. Myosin heavy chain isoform expression in adult and juvenile mini-muscle mice bred for high-voluntary wheel running. Mech Develop 2014;134:16-30.

26. Gray H. Anatomy of the human body. Philadelphia: Lea \& Febiger, 1918. Bartleby.com, 2000; 20th. Available from: http://www.bartleby.com/107/illus 432.html

27. Koutakis P, Miserlis D, Myers SA, Kim JK, Zhu Z, Papoutsi E, et al. Abnormal accumulation of desmin in gastrocnemius myofibers of patients with peripheral artery disease: associations with altered myofiber morphology and density, mitochondrial dysfunction and impaired limb function. J Histochem Cytochem 2015;63: 256-69.

28. Walmsley GL, Blot S, Venner K, Sewry C, Laporte J, Blondelle J, et al. Progressive structural defects in canine centronuclear myopathy indicate a role for HACD1 in maintaining skeletal muscle membrane systems. Amn J Pathol. 2017;187:441-56.

29. Prats C, Gomez-Cabello A, Nordby P, Andersen JL, Helge JW, Dela F, et al. An optimized histochemical method to assess skeletal muscle glycogen and lipid stores reveals two metabolically distinct populations of type I muscle fibers. PLoS One 2013;8:e77774.

30. Sawano S, Komiya Y, Ichitsubo R, Ohkawa Y, Nakamura M, Tatsumi R, et al. A one-step immunostaining method to visualize rodent muscle fiber type within a single specimen. PLoS One 2016;11: e0166080.

31. Dyar KA, Ciciliot S, Wright LE, Bienso RS, Tagliazucchi GM, Patel VR, et al. Muscle insulin sensitivity and glucose metabolism are controlled by the intrinsic muscle clock. Mol Metab 2014;3:29-41. 